\title{
The Predictive Power of Omics: Clinical
} Applications

\section{Moushumi Nath, ${ }^{a}$ Xinwen Zhu ${ }^{\mathrm{b}}$}

aDepartment of Physiology, Faculty of Medicine, University of Toronto.

bepartment of Pharmacology and Therapeutics, Faculty of Medicine, McGill University.

*Selected as Top Article

\section{Introduction}

Scientia potentia est: "knowledge is power."

This simple idea drives medical decision-making. To maintain the health and well-being of their patients, physicians must acquire information on environmental and biological factors that may predict disease risk, progression, and treatment effectiveness. However, traditional clinical tools are limited in their ability to capture information, usually relying on only a handful of readouts. For example, questionnaires or blood tests can be used to reveal certain parameters, such as anxiety level or red blood cell count, but not the totality of the condition. Enter the "omics" revolution: the advent of technologies that aim to capture complete information about some aspect of physiological systems. These omics technologies, including various high-throughput DNA and RNA sequencing platforms, as well as mass spectrometry analyses of protein and metabolite levels, allow for the holistic consideration of the complex networks and interactions that form and sustain each individual $(1,2)$. Patientspecific data can be used in computational models that predict disease onset, disease progression, and treatment effectiveness, thus facilitating medical decisionmaking (Figure 1) $(3,4)$. The value of omics in healthcare is therefore the predictive power it provides.

\section{The Predictive Power of Omics in: Disease Risk and Onset}

Omics can be applied to assess both disease risk and onset. Genomics, for example, can be used to predict the lifetime risk of a disease. Currently, physicians collect information on family history and may screen for a select number of genetic risk factors. In contrast, genomics has the potential to identify all possible genetic risk factors for any disease (3). This increases the likelihood of capturing rarer genetic risk factors or genetic variants that increase risk to lesser extents, providing a more accurate risk assessment. Additionally, other omics technologies could be used to predict disease onset $(5,6)$. The power of the combined omics analysis was demonstrated by the Snyder Lab at Stanford University. An integrative Personal Omics Profile (iPoP) was produced for one individual over the course of 14 months through recurrent measurements of transcriptomics, proteomics, and metabolomics. This study identified that the subject had an elevated risk of developing diabetes, and observed increases in blood glucose concentrations that signaled the onset of the disease; following diet changes, exercise, and low doses of aspirin, the glucose levels returned to normal (5). Regular monitoring of generally healthy individuals with omics-based technologies

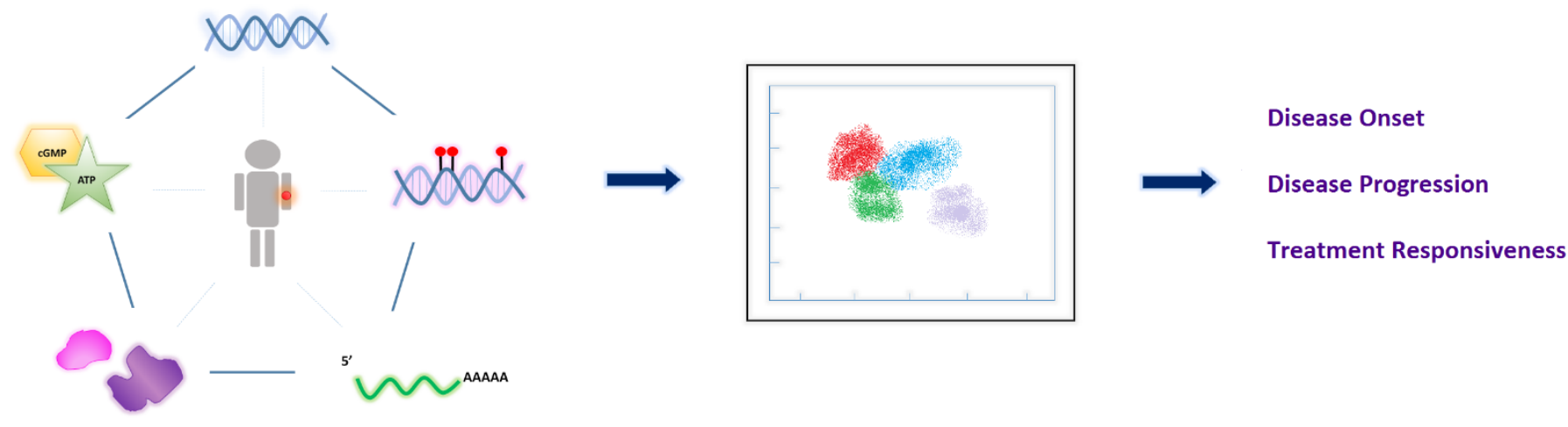

Figure I: Information acquired through different omics technologies (genomics, epigenomics, transcriptomics, proteomics, metabolomics, and more) can be used in computational models to accurately predict different health parameters. 
- could generate data that herald the onset of disease, allowing early diagnosis and early intervention, which are both crucial to treatment success.

\section{Prognoses}

Prognostic factors include progression of disease severity, likelihood of survival, and survival time. Accurate predictions of these factors have important roles in medical decision-making, affecting treatment and end-of-life decisions (7). One omics-based test currently applied in making prognoses is the Oncotype DX test (2). This test examines the gene expression profile of a patient's breast cancer tumour in order to predict the likelihood of breast cancer recurrence, information that can aid in treatment selection. Individuals with low risk of recurrence are treated with endocrine therapy alone. In contrast, individuals with high risk of recurrence are treated with both endocrine therapy and chemotherapy. This way, individuals with low risk of recurrence are pre-empted from unnecessary and highly invasive treatments with minimal therapeutic effects. A meta-analysis of medical decision-making reveals that more than $1 / 3$ of physicians have omitted chemotherapy in the treatment of breast cancer patients based on results obtained from the Oncotype DX test (2). Therefore, omics can assist physicians and patients in making informed decisions by facilitating accurate prognoses.

\section{Treatment Effectiveness}

Not all treatments are equally effective for all patients; what cures one individual might only cause adverse side effects in another individual with an ostensibly similar ailment. Omics-based techniques can help healthcare professionals formulate optimal treatment strategies for each patient by allowing for better characterization of the biochemical underpinnings of diseases and improved prediction of the effects of pharmacological intervention (8). Genomic sequencing of tumours can identify the exact mutations responsible for an individual's cancer, so that the appropriate pathways may be targeted. Selection of drugs for treatment should be guided by pharmacogenomics, a relatively new field whose development is based on the availability and continued improvement of genome sequencing techniques. Pharmacogenomics examines how genetic differences between individuals underlie differential responses to drugs. For instance, dosing strategies for commonly used drugs such as warfarin are adjusted according to genetic polymorphisms affecting the patient's drug-metabolizing enzymes (9). Even more recently, developments in epigenomics have sparked interest in the modulation of drug effects by epigenetic mechanisms. Knowing a patient's genetic and epigenetic background will therefore be crucial to picking treatment strategies that are most likely to succeed.

\section{Limitations and Conclusions}

Current barriers to the implementation of omics approaches in clinical practice are mainly logistical in nature. Although powerful, many of the methods of data collection and analysis are time consuming and resource-intensive. Conducting reliable analyses of these large data sets is an additional hurdle, as the study of systems and computational biology is still in its infancy and the processing of omics data is computationally intensive (8). Nevertheless, as the costs of omics technologies decrease and more holistic research is conducted, current trends suggest the eventual widespread use of omics in personalized medicine (3).

The ability of omics to capture the totality of one aspect of physiological systems in a hypothesis-free manner facilitates the accurate prediction of disease onset, progression, and treatment responsiveness. This high predictive power sets it apart from traditional methods of treatment that require a number of guessand-check points. Omics will be an important influence on the healthcare system, necessitating large collaborative efforts. These efforts will yield highly accurate healthcarerelated prediction models, which will have tremendous influence on medical decision-making and individual healthcare.

\section{References}

1. McShane LM, Cavenagh MM, Lively TG, Eberhard DA, Bigbee WL, Williams PM, et al. Criteria for the use of omics-based predictors in clinical trials. Nature. 2013;502(7471):317-320.

2. Micheel CM, Nass SJ, Omenn GS. Evolution of Translational Omics : Lessons Learned and the Path Forward. Washington, US: National Academies Press; 2012.

3. Rehm HL. Evolving health care through personal genomics. Nat Rev Genet. 2017; advance online publication.

4. Simon R, Roychowdhury S. Implementing personalized cancer genomics in clinical trials. Nat Rev Drug Discov. 2013;12(5):35869.

5. Chen R, Mias George I, Li-Pook-Than J, Jiang L, Lam Hugo YK, Chen R, et al. Personal omics profiling reveals dynamic molecular and medical phenotypes. Cell. 2012;148(6):1293-307.

6. Wishart DS. Emerging applications of metabolomics in drug discovery and precision medicine. Nat Rev Drug Discov. 2016;15(7):473-84.

7. Tebani A, Afonso C, Marret S, Bekri S. Omics-based strategies in precision medicine: Toward a paradigm shift in inborn errors of metabolism investigations. Int J Mol Sci. 2016;17(9).

8. Chen R, Snyder M. Promise of personalized omics to precision medicine. Wiley Interdiscip Rev Syst Biol Med. 2013;5(1):73-82.

9. Mega JL, Giugliano RP. Genotype-guided dosing of warfarin. Clin Chem. 2014;60(7):920-922. 

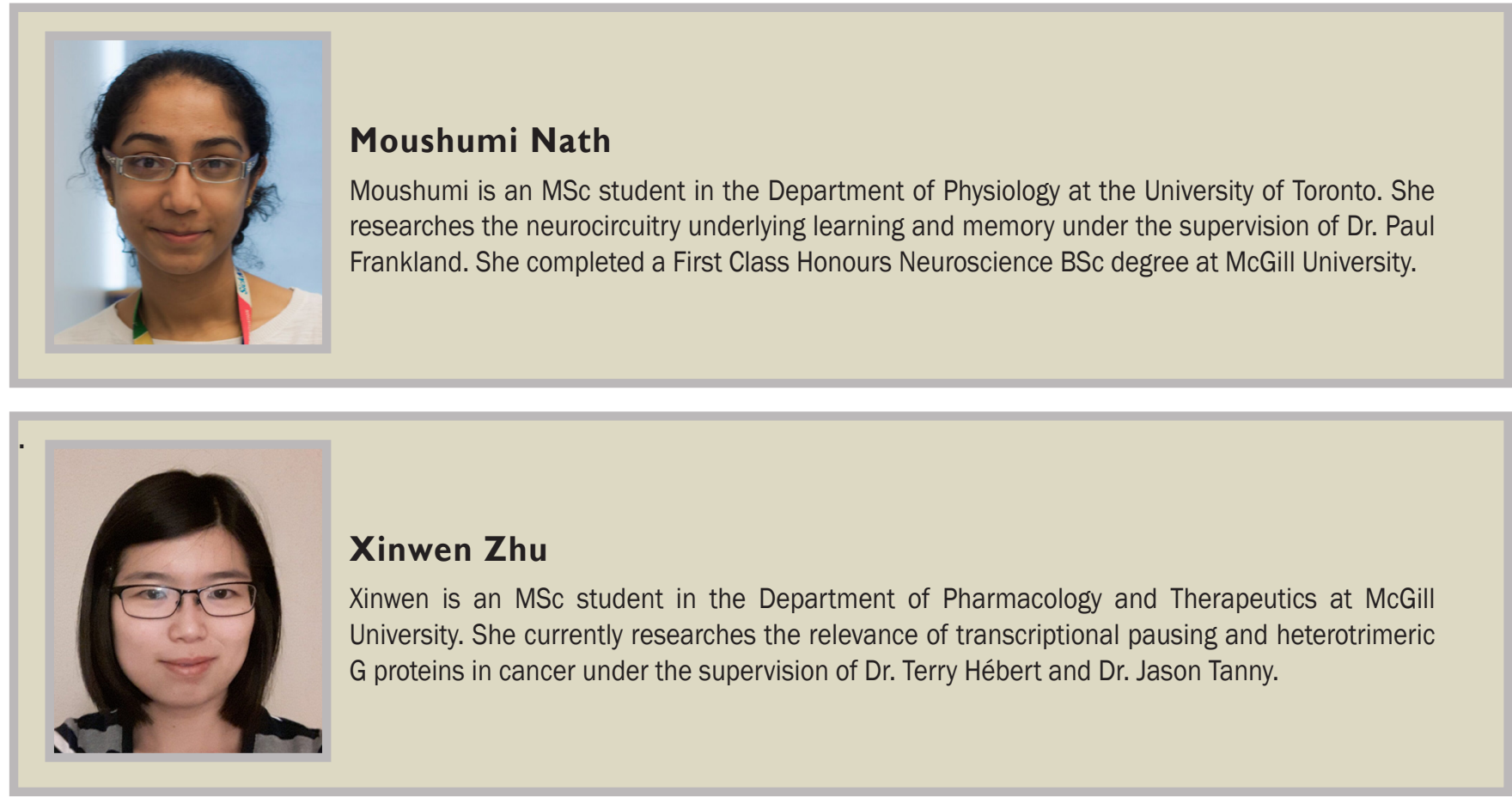